\title{
The Dissociative Subtype of Posttraumatic Stress Disorder: Unique Resting-State Functional Connectivity of Basolateral and Centromedial Amygdala Complexes
}

\author{
Andrew A Nicholson', Maria Densmore ${ }^{2}$, Paul A Frewen ${ }^{3}$, Jean Théberge ${ }^{4}$, Richard WJ Neufeld $^{5}$, \\ Margaret C McKinnon ${ }^{6}$ and Ruth A Lanius*,3 \\ 'Schulich School of Medicine and Dentistry, Western University, London, Ontario, Canada; 'Lawson Health Research Institute, London, Ontario, \\ Canada; ${ }^{3}$ Lawson Health Research Institute, Western University, London, Ontario, Canada; ${ }^{4}$ Department of Diagnostic Imaging, St Joseph's Health \\ Care London, Departments of Medical Imaging, Medical Biophysics and Psychiatry, Western University, London, Ontario, Canada; ${ }^{5}$ Departments of \\ Psychology and Psychiatry, Neuroscience Program, Western University, London, Ontario, Canada; ${ }^{6}$ McMaster University, St Joseph's Healthcare \\ Hamilton, Homewood Research Institute, Hamilton, Ontario, Canada
}

\begin{abstract}
Previous studies point towards differential connectivity patterns among basolateral (BLA) and centromedial (CMA) amygdala regions in patients with posttraumatic stress disorder (PTSD) as compared with controls. Here we describe the first study to compare directly connectivity patterns of the BLA and CMA complexes between PTSD patients with and without the dissociative subtype (PTSD+DS and PTSD - DS, respectively). Amygdala connectivity to regulatory prefrontal regions and parietal regions involved in consciousness and proprioception were expected to differ between these two groups based on differential limbic regulation and behavioral symptoms. PTSD patients $(n=49)$ with $(n=13)$ and without $(n=36)$ the dissociative subtype and age-matched healthy controls $(n=40)$ underwent restingstate $\mathrm{fMRI}$. Bilateral BLA and CMA connectivity patterns were compared using a seed-based approach via SPM Anatomy Toolbox. Among patients with PTSD, the PTSD+DS group exhibited greater amygdala functional connectivity to prefrontal regions involved in emotion regulation (bilateral BLA and left CMA to the middle frontal gyrus and bilateral CMA to the medial frontal gyrus) as compared with the PTSD - DS group. In addition, the PTSD+DS group showed greater amygdala connectivity to regions involved in consciousness, awareness, and proprioception -implicated in depersonalization and derealization (left BLA to superior parietal lobe and cerebellar culmen; left CMA to dorsal posterior cingulate and precuneus). Differences in amygdala complex connectivity to specific brain regions parallel the unique symptom profiles of the PTSD subgroups and point towards unique biological markers of the dissociative subtype of PTSD.

Neuropsychopharmacology (2015) 40, 2317-2326; doi:I0.I038/npp.20I5.79; published online 22 April 2015
\end{abstract}

\section{INTRODUCTION}

The dissociative subtype of posttraumatic stress disorder (PTSD+DS)-exhibited by $13-30 \%$ of PTSD patients-has recently been added to the fifth revision of the Diagnostic and Statistical Manual (American Psychiatric Association, 2013; Lanius et al, 2012; Stein et al, 2013; Steuwe et al, 2012; Wolf et al, 2012). In addition to genetic predispositions associated with the emergence of PTSD+DS (Wolf et al, 2014), epidemiological evidence points to several risk factors also associated with PTSD+DS, including prior trauma, childhood adversities, and childhood onset of PTSD (Stein et al, 2013).

\footnotetext{
*Correspondence: Dr RA Lanius, Department of Psychiatry, London Health Sciences Centre-University Hospital, 339 Windermere Road, Room C3-103, PO Box 5339, London, Ontario N6A 5A5, Canada, Tel: +519 663 3306, E-mail: Ruth.lanius@|hsc.on.ca Received I October 2014; revised 10 March 2015; accepted 16 March 2015; accepted article preview online 19 March 2015
}

Numerous studies point towards differential patterns of neural activation of the amygdala, prefrontal cortex (PFC), anterior cingulate cortex (ACC), precuneus, and parietal lobe in PTSD patients who exhibit dissociative responses during traumatic script-driven imagery or fear processing as compared with those who instead demonstrate re-experiencing symptoms (Felmingham et al, 2008; Hopper et al, 2007; Lanius et al, 2002; 2005; 2010a). This observation led to the identification of two types of emotion and amygdalar dysregulation among PTSD patients, proposed to occur at baseline as well as in response to trauma reminders: undermodulation and overmodulation (Lanius et al, 2010b; 2012). Emotional undermodulation consists of reliving traumatic experiences with related hyperarousal, characteristic of non-dissociative PTSD patients (PTSD-DS). This behavioral response is associated with decreased activation of PFC regions and ACC, thereby decreasing top-down inhibition of the amygdala and leading to hyperactivation of the limbic system (Lanius et al, 2010b; 2012; Shin et al, 2005; Shin and Liberzon, 2010; also see Birn et al, 2014; Stevens et al, 2013; Yan et al, 2014). 
Here, Lévesque et al (2003) report increased activation within the right dorsolateral prefrontal cortex (dlPFC) when healthy participants were asked to suppress negative emotions that were associated with increased amygdala activity. Similarly, Banks et al (2007) show that the dlPFC exhibits connectivity to the amygdala when healthy participants were asked to regulate negative affect, suggesting top-down PFC inhibition of the amygdala. A recent study has also reported that hyperarousal is associated with negative mPFCamygdala coupling in PTSD (Sadeh et al, 2014). In contrast to emotional undermodulation, overmodulation is associated with emotional detachment and hypoarousal involving symptoms of depersonalization and derealization characteristic of PTSD+DS. Emotional overmodulation is thought to be mediated by increased PFC and ACC activation, resulting in excessive top-down inhibition of the amygdala (Lanius et al, 2010b; 2012). Right amygdala activity has also been shown to be negatively correlated with trait dissociative symptoms during exposure to thermal pain in PTSD patients (Mickleborough et al, 2011). Additionally, findings from a meta-analysis examining neuroimaging findings in PTSD have demonstrated a dorsal hypoactive amygdala region, comprising the centromedial complex (CMA; see below), which was suggested by the authors to be related to autonomic blunting associated with emotional numbing and dissociation (Etkin and Wager, 2007). Taken together, these findings suggest that the amygdala may exhibit differential neuromodulation in PTSD+DS and PTSD - DS.

The amygdala can be classified into two subdivisions, forming the basolateral (BLA) and CMA amygdala complexes. The BLA and CMA have differential patterns of functional and structural connectivity, in addition to unique roles in fear processing (Roy et al, 2009; Phelps et al, 2004), which are altered in PTSD patients (Milad, 2014; Sripada et al, 2012). The BLA evaluates sensory information and mediates cortical integration of fear and other emotions (Jovanovic and Ressler, 2010). The BLA is regulated by feedforward inhibition from the medial PFC via somatostatin connections, with outputs to the thalamus, striatum, and PFC (Duvarci and Pare, 2014). The CMA is involved in the execution of fear responses, with GABAergic outputs to the brainstem and periaqueductal gray involved in descending pain modulation (Duvarci and Pare, 2014; LeDoux, 1998; Milad, 2013). The CMA also receives thalamic projections and mediates major nociceptive pathways (Duvarci and Pare, 2014). Notably, deactivation of the CMA results in the impairment of fear expression and acquisition (Duvarci and Pare, 2014).

A novel study by Brown et al (2014) reported differential resting-state connectivity of the BLA but not the CMA between military-trauma exposed PTSD patients and veteran controls, where the PTSD group showed greater connectivity to the pregenual ACC, dorsomedial PFC, and dorsal ACC and controls showed stronger connectivity to the left inferior frontal gyrus. These findings suggest that the BLA and CMA complexes may exhibit unique roles in relation to PTSD symptomatology.

Critically, no studies have compared directly patterns of amygdala complex (BLA and CMA) resting-state connectivity in PTSD+DS vs PTSD - DS, which is the objective of the current study. Due to the proposed differential PFC- and ACC-mediated inhibition of the amygdala in PTSD+DS and PTSD-DS (exacerbated inhibition and attenuated inhibition, respectively), connectivity between the BLA and
PFC (dlPFC) and ACC was expected to be greater in the PTSD+DS as compared with the PTSD - DS group. Moreover, given that patients with PTSD+DS and PTSD-DS exhibit unique behavioral symptoms, connectivity of the CMA was predicted to differ between these groups. Finally, we hypothesized altered amygdala connectivity (BLA and CMA) to lateral and midline parietal regions involved in consciousness, awareness, and proprioception among PTSD patients-which may contribute to the unique symptoms observed between the two patient groups.

\section{MATERIALS AND METHODS}

\section{Participants}

Participants were recruited between 2009 and 2013 from the Department of Psychiatry London Health Services Center (LHSC), through family physicians, mental health professionals, psychology/psychiatric clinics, community programs for traumatic-stress survivors, and posters/advertisements, all within the London, ON community. The sample consisted of 49 PTSD patients and 40 age-matched healthy controls (demographic and clinical information are summarized in Table 1). Exclusion criteria for all participants included: any implants or metal that do not comply with $3 \mathrm{~T}$ fMRI safety standards, a history of head injury with any loss of consciousness, significant untreated medical illness, a history of neurological disorders, a history of any pervasive developmental disorders, pregnancy, and current use of any psychotropic or cardiovascular medications within 1 month prior to the study. The exclusion criteria for PTSD patients included: history of bipolar disorder or schizophrenia, and alcohol or substance dependence/abuse not in sustained full remission within 6 months prior to participation in the study. Exclusion criteria for the control group consisted of current or past Axis-I or Axis-II disorders. Participants were assessed using the DSM-IV Structured Clinical Interview (SCID) (First, 1997), the Clinical Administered PTSD Scale (CAPS) (Blake et al, 1995), Beck's Depression Inventory (BDI) (Beck et al, 1997), and the Childhood Trauma Questionnaire (CTQ) (Bernstein et al, 2003). Additionally, the Multiscale Dissociation Inventory (MDI) (Briere et al, 2005) was administered, which is a standard 30-item test of 6 types of dissociative responses: Disengagement (DENG), Depersonalization (DEPR), Derealization (DERL), Emotional Constriction (ECON), Memory Disturbance (MEMD), and Identity Dissociation (IDDIS). All PTSD participants met criterion A in the DSM-IV and had a CAPS score of $>50$. To be classified as PTSD+DS, PTSD patients required a score of at least two on both frequency and intensity for either the CAPS depersonalization or derealization items, a conservative standard method of DSM-IV PTSD+DS characterization (Steuwe et al, 2012), which restricts false positives. All scanning took place at the Robarts Research Institute's Center for Functional and Metabolic Mapping in London, Ontario, Canada. The research ethics board at the University of Western Ontario approved the current study, and all participants provided written informed consent.

\section{fMRI Data Acquisition}

Functional images were collected using a $3.0 \mathrm{~T}$, whole-body MRI scanner (Magnetom Tim Trio, Siemens Medical 
Table I Demographic and Clinical Information

\begin{tabular}{|c|c|c|c|}
\hline Measure & $\begin{array}{c}\text { PTSD - DS } \\
(n=36)\end{array}$ & PTSD+DS $(n=13)$ & $\begin{array}{l}\text { Controls } \\
(n=40)\end{array}$ \\
\hline Age & $M=37 \pm 12.9$ & $M=37 \pm 12.7$ & $M=32.3 \pm 11.4$ \\
\hline Sex & Females $=26$ & Females $=1 \mathrm{I}$ & Females $=29$ \\
\hline CAPS & $69.0 \pm 13.2$ & $79.5 \pm 20.2$ & $0.7 \pm 3.0$ \\
\hline CTQ & $63.0 \pm 25.9$ & $71.2 \pm 20.2$ & $31.6 \pm 8.4$ \\
\hline BDI & $24.3 \pm 8.1$ & $33.5 \pm 11.6$ & $1.6 \pm 2.6$ \\
\hline MDI-DENG & $14.0 \pm 5.3$ & $17 \pm 4.6$ & $8.0 \pm 2.4$ \\
\hline MDI-DEPR & $7.0 \pm 3.2$ & $12.3 \pm 4.5$ & $5.3 \pm 0.7$ \\
\hline MDI-DERL & $9.0 \pm 3.7$ & $13.8 \pm 4.0$ & $5.3 \pm 0.7$ \\
\hline MDI-ECON & $11.0 \pm 5.6$ & $17.25 \pm 5.3$ & $5.3 \pm 0.8$ \\
\hline MDI-MEMD & $9 \pm 3.7$ & $14.8 \pm 4.0$ & $5.7 \pm 1.1$ \\
\hline MDI-DDIS & $6 \pm 2.3$ & $9.4 \pm 5.1$ & $5.0 \pm 0.0$ \\
\hline MDD & $n=1$ & $n=1$ & $n=0$ \\
\hline Panic disorder & $n=1$ & $n=0$ & $n=0$ \\
\hline Agoraphobia & $n=0$ & $n=0$ & $n=0$ \\
\hline Social phobia & $n=0$ & $n=2$ & $n=0$ \\
\hline OCD & $n=0$ & $n=0$ & $n=0$ \\
\hline GAD & $n=0$ & $n=0$ & $n=0$ \\
\hline Somatization disorder & $n=0$ & $n=0$ & $n=0$ \\
\hline Somatoform disorder & $n=0$ & $n=1$ & $n=0$ \\
\hline Eating disorders & $n=0$ & $n=0$ & $n=0$ \\
\hline
\end{tabular}

Abbreviations: BDI, Becks Depression Inventory; CAPS, Clinician Administered PTSD Scale; Control, age-matched control group; CTQ, Childhood Trauma Questionnaire; DENG, Disengagement; DEPR, Depersonalization; DERL, Derealization; ECON, Emotional Constriction; GAD, Generalized Anxiety Disorder; IDDID, Identity Dissociation; MDD, Major Depressive Disorder; MDI, Multiscale Dissociation Inventory; MEMD, Memory Disturbance; n, number of participants corresponding to a group; $O C D$, Obsessive Compulsive Disorder; PTSD+DS, dissociative subtype PTSD group; PTSD - DS, non-dissociative PTSD group.

Solutions, Erlangen, Germany) with the manufacturer's 32-channel phased array head coil. BOLD fMRI images were acquired with the manufacturer's standard gradient-echo EPI pulse sequence (single-shot, blipped-EPI, interleaved slice acquisition order and tridimensional prospective acquisition correction). Participants' heads were immobilized with foam padding within the head coil. EPI volumes were acquired with $2 \mathrm{~mm}$ isotropic resolution with the following parameters: $\mathrm{FOV}=192 \times 192 \times 128 \mathrm{~mm}^{3} \quad(94 \times 94$ matrix, 64 slices), $\mathrm{TR} / \mathrm{TE}=3000 \mathrm{~ms} / 20 \mathrm{~ms}$, flip angle $=90^{\circ}, 120$ volumes. Participants were asked to close their eyes, relax, and let their minds wander during the 6-min (120-volume) scan. After the scan, participants were asked whether they had been able to comply with the instructions and whether they felt as though they had drifted out of wakefulness (all participants had been able to comply with the instructions, and no participant reported having drifted out of wakefulness).

\section{fMRI Data Preprocessing}

Image preprocessing and statistical analyses were conducted using Statistical Parametric Mapping (SPM8 and SPM12, Wellcome Department of Neurology, London, UK: http:// www.fil.ion.ucl.ac.uk/spm) within Matlab 8.3 (The Mathworks Inc., MA). For each participant, all functional images were realigned to the first image in the series, resliced, and mean functional images were created. Functional images were further motion corrected using the ART software (Gabrieli Lab. McGovern Institute for Brain Research, Cambridge, MA), which computes motion outlier regressors to be used in the first-level analysis as a covariate of no interest. Movement did not differ between participant groups based on SPM and ART parameters. The images were then spatially normalized. The mean image was co-registered to the SPM EPI template, and the resulting deformation matrix was applied to the functional images. All images were then smoothed using a 6 $\mathrm{mm}$ full-width-half-maximum isotropic Gaussian filter. Band-pass filtering was conducted using successive application of a high-pass and low-pass filter with frequency cutoffs of 0.012 and $0.1 \mathrm{~Hz}$ respectively (in-house software by coauthor Jean Théberge). Seed region-of-interest masks were created using SPM's Anatomy Toolbox (Eickhoff et al, 2005) featuring cytoarchitectonically based probability maps of the amygdala. Connectivity correlations were standardized using a Fisher $Z$ transformation in SPM8.

\section{Statistical Analyses}

Subject-level analyses were conducted separately for each amygdala ROI. For each participant, a mean signal intensity time course was extracted from SPM's Anatomy Toolbox for each of the four seed regions (bilateral BLA and bilateral CMA) and then used as a regressor in a correlation analysis with the whole-brain resting scan data for that participant. Connectivity is thus indicative of a correlation between amygdala seed regions and other brain areas. Both positive correlations and anticorrelations were examined. A wholebrain 3 (group) $\times 2$ (complex) full-factorial analysis of variance (ANOVA) was conducted in the second-level analyses. The between-group factor consisted of three levels: PTSD+DS, PTSD-DS, and controls, and the within-group factor consisted of two levels: BLA and CMA. The variance for the between-group factor was set to unequal in order to account for the unequal sample size (Milligan et al, 1987). Here a restricted maximum likelihood adjustment was made to the degrees of freedom during inference, using weighted least squares to produce Gauss-Markov estimators. The group $\times$ complex interaction yielded eight significant (FWEcorrected $p<0.05, k=10$ ) gray matter clusters (see Table 2 for ANOVA results). Follow-up comparisons for the eight coordinates meeting the above error rate protection were then conducted examining connectivity values in one- and two-sample $t$-tests. Follow-up analyses utilized $p$-uncorrected $<0.005 k=10$, following the suggestion of Lieberman and Cunningham (2009) to balance the relative risk of Type I vs II error rates. One-sample within-group analyses were conducted for each PTSD patient group and control group, individually for each amygdala seed region. Two-sample between-group contrasts were explored for each seed region, comparing both PTSD patient groups, and each patient group to the control group. Similarily, bilateral dlPFC coordinates were specified a-priori and used to conduct small volume correction analyses with an 8 - $\mathrm{mm}$ radius sphere (MNI 36, 25, 30: Lévesque et al, 2003; MNI 48, 28, 36; and MNI - 12, 22, 60: Banks et al, 2007) (FWE-corrected 
Table 23 (Group) $\times 2$ (Complex) Full Factorial ANOVA

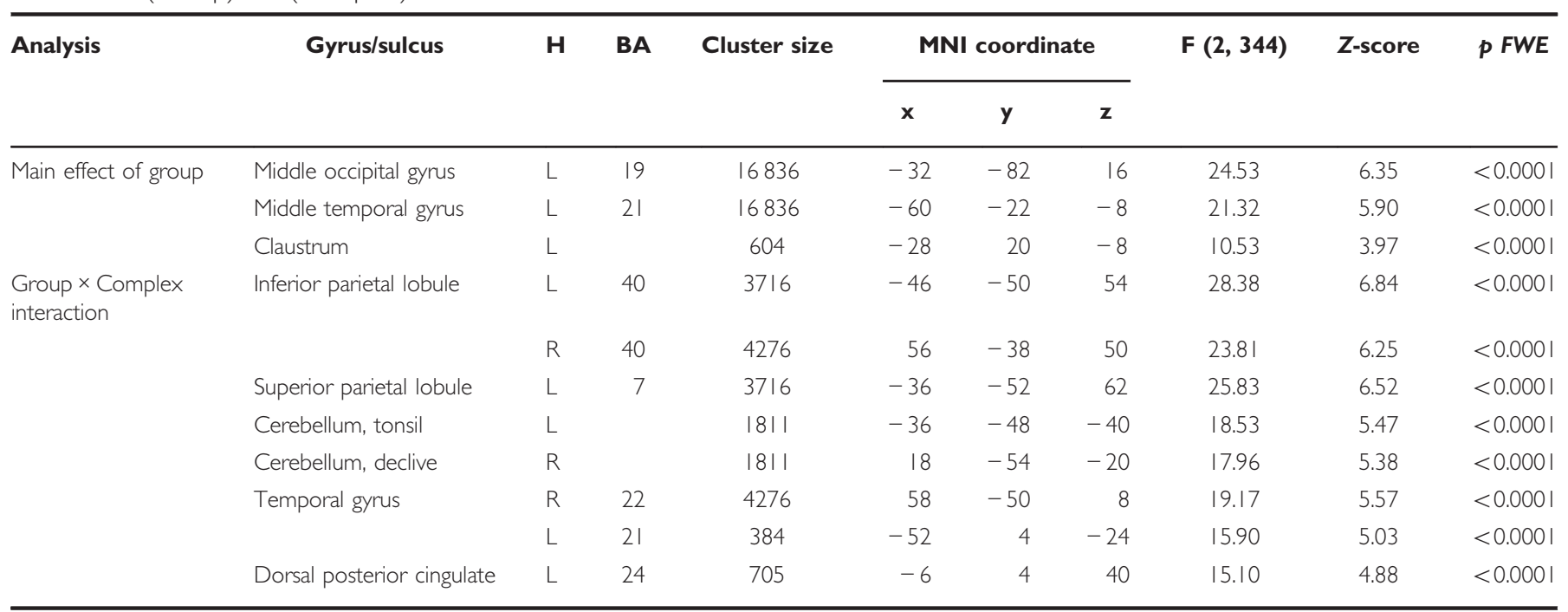

Abbreviations: BA, Broadmann area; FWE, family-wise error cluster-corrected threshold; $\mathrm{H}$, hemisphere. Full factorial analysis of variance displaying FWE-corrected gray matter clusters.

$p<0.05 k=10)$. This region was chosen as the model postulated by Lanius et al (2010b) and hypothesizes that PFC regions exhibit differential top-down inhibition in PTSD+DS vs PTSD - DS. Critically, Lévesque et al (2003) report increased activation within the right dlPFC when participants were asked to voluntarily suppress negative emotion-where negative emotion was associated with increased amygdala activation. Banks et al (2007) show that bilaterally the dlPFC exhibits connectivity to the amygdala when participants were asked to regulate negative affect.

Baseline amygdala activity was also compared across groups for each seed to avoid potential confounding factors when drawing inferences about activity correlations. This is important as differences in connectivity between groups may be driven by the unique baseline activity of the seed region (see Supplementary Methods). To measure baseline amygdala complex activity, mean signal intensity time courses were extracted for each amygdala seed on the participant level to compare across groups. In addition, clinical variables CAPs, CTQ, MDI, and BDI were compared across groups. One-way independent ANOVAs were used to compare baseline amygdala activity and clinical variables (see Supplementary Methods for statistics protocol). Furthermore, subscale MDI depersonalization and derealization items were averaged for each participant and evaluated as a predictor of amygdala connectivity in a multiple regression, including data from all PTSD (PTSD - DS and PTSD+DS) patients, examining the eight FWE-corrected clusters reported from the group $\times$ complex interaction and coordinates from the dlPFC a-priori region-of-interest.

\section{RESULTS}

\section{Clinical Variables and Baseline Amygdalar Activity}

All clinical variables (CAPS, BDI, CTQ, and MDI) yielded significant values for Levene's test of homogeneity of variance (means and SDs are presented in Table 1, statistics are presented in Table 3). Via Games-Howell comparisons, no significant differences were found between the PTSD+DS and PTSD - DS groups with regard to CAPS, BDI, and CTQ scores. MDI scores were significantly higher in the PTSD + DS as compared with the PTSD - DS group. MDI and CTQ scores were higher in the PTSD+DS and PTSD - DS groups as compared with the control group. There were no betweenpatient group differences in baseline amygdala activity (see Supplementary Results: Supplementary Table S5 for means and SDs, Supplementary Table S6 for statistical details) and, therefore connectivity comparisons between patient groups could be interpreted with confidence in this regard.

\section{One-Sample Functional Connectivity Within the Dissociative Subtype, Non-Dissociative PTSD and Control Groups}

Resting-state activity in the BLA and CMA predicted widespread activation in multiple cortical and subcortical regions within all the participant groups for both the coordinates extracted from the ANOVA interaction and the a-priori dlPFC region. These results generally replicated findings by Brown et al, (2014) and Roy et al, (2009). As they are not the main focus of the current study, within-group data are reported in Supplementary Results (see Supplementary Tables S1). There were no significant anticorrelations for within-group connectivity maps.

\section{Dissociative Subtype and Non-dissociative PTSD Group Differences in Functional Connectivity}

When examining connectivity values from the ANOVA interaction coordinates, the PTSD+DS group showed greater functional connectivity between the left BLA and the left superior parietal lobe (SPL) (BA 5) and the left culmen of the cerebellum (Figure 1a; Table 4a) as compared with the PTSD - DS group. The PTSD+DS also showed greater functional connectivity between the left CMA and the left dorsal posterior cingulate (BA 24), left medial frontal gyrus (BA6), and the left precuneus (BA 7) (Figure 1c), while the 
Table 3 Clinical Variables

\begin{tabular}{|c|c|c|c|c|c|}
\hline Variable & Levene's homogenity test & ANOVA & Post-hoc comparisons & Tukey-Kramar HSD & Games-Howell \\
\hline CAPS & $F(2,86)=36.067, p<0.001$ & NA & PTSD - DS and PTSD+DS & NA & $t(3,22)=2.473, \mathrm{NS}$ \\
\hline BDI & $F(2,81)=|7.439, p<0.00|$ & NA & PTSD - DS and PTSD+DS & NA & $t(3,15)=2.530, \mathrm{NS}$ \\
\hline \multirow[t]{3}{*}{ CTQ } & $F(2,86)=19.499, p<0.001$ & NA & PTSD - DS and PTSD+DS & NA & $t(3,27)=1.475, \mathrm{NS}$ \\
\hline & & & PTSD - DS and Control & NA & $t(3,4 I)=6.93, p<0.001$ \\
\hline & & & PTSD+DS and Control & NA & $t(3,13)=6.880, p<0.001$ \\
\hline \multirow{2}{*}{ MDI Total } & & & PTSD - DS and Control & NA & $t(3,38)=7.250, p<0.001$ \\
\hline & & & PTSD+DS and Control & NA & $t(3,34)=9.238, p<0.001$ \\
\hline
\end{tabular}

Abbreviations: BLA, basolateral amygdala baseline activity; BDI, Becks Depression Inventory; CAPS, Clinician Administered PTSD Scale; CMA, Centromedial amygdala baseline activity; Control, age-matched control group; CTQ, Childhood Trauma Questionnaire; MDI, Multiscale Dissociation Inventory; NA, not applicable; PTSD+DS, dissociative subtype PTSD group; PTSD - DS, non-dissociative PTSD group.

right CMA showed greater functional connectivity to the left medial frontal gyrus (BA 32) (Figure 1d) as compared with the PTSD - DS group. The PTSD - DS group did not show any significantly greater connectivity patterns to any of the amygdala complexes as compared with the PTSD+DS group.

In addition, when examining the dlPFC a-priori region, the PTSD+DS group showed greater functional connectivity between both the left and right BLA and the right middle frontal gyrus (BA 9) (Figure 1a and b; Table 4b). The PTSD + DS group also showed greater connectivity between the left CMA and the right middle frontal gyrus (BA 9) (Figure 1c).

\section{Correlations to Functional Connectivity Within PTSD Patients}

Higher depersonalization and derealization MDI scores predicted increased connectivity between the left BLA and right middle frontal gyrus - a region showing one of the strongest between-patient group differences in connectivity in the main analysis (see Table 5).

\section{Control Group and PTSD Patient Group Differences in Functional Connectivity}

When investigating between-group BLA and CMA functional connectivity, widespread cortical and subcortical differences were found when comparing the control group to both the PTSD+DS and PTSD - DS groups for both the coordinates extracted from the ANOVA interaction and a-priori dlPFC region. Control group comparisons are similarly reported in Supplementary Results (see Supplementary Table S4).

\section{DISCUSSION}

The primary aim of the present study was to compare connectivity patterns of the BLA and CMA complexes between individuals exhibiting PTSD+DS and PTSD - DS. As compared with the PTSD - DS group, the PTSD+DS group exhibited greater amygdala functional connectivity to prefrontal regions involved in emotion regulation (bilateral BLA and left CMA to the middle frontal gyrus and bilateral CMA to the medial frontal gyrus). In addition, the PTSD+DS group showed greater amygdala connectivity to regions involved in consciousness, awareness, and proprioception (left BLA to superior parietal lobe and cerebellar culmen; left CMA to dorsal posterior cingulate and precuneus) as compared with the PTSD - DS group.

In keeping with our hypothesis, we found increased functional connectivity between the amygdala and PFC regions in PTSD+DS as compared with PTSD-DS. This finding is in line with the proposed increased $v s$ decreased PFC inhibition of limbic regions in PTSD+DS $v s$ PTSD - DS, respectively (Lanius et al, 2010b). There was also greater connectivity in the PTSD+DS group between both bilateral BLA and left CMA to the right middle frontal gyrus and between bilateral CMA and the left medial frontal gyrus as compared with the PTSD - DS group. This parallels previous findings in terms of structural connectivity of the BLA, which projects outputs to the PFC and receives feedforward somatostatin inhibition from cortical inputs (Duvarci and Pare, 2014). Etkin et al (2011) suggest that there is an imperfect separation of function in ventral and lateral PFC with regard to appraisal/expression and regulation of emotion, respectively. Group differences in amygdala connectivity with the middle and medial frontal gyri in the present study may therefore underlie emotional appraisal/ expression and/or regulation. Previously, Lévesque et al (2003) also report that the lateral portion of the right dlPFC is associated with the voluntary suppression of negative emotion when healthy participants were asked to suppress sadness, where increased sadness was associated with activation in the amygdala. In a connectivity analysis by Banks et al (2007), prefrontal dorsolateral and dorsomedial cortices, as well as ACC, showed increased connectivity to the amygdala when healthy participants were asked to regulate their negative affect, suggesting top-down inhibition of the amygdala. Notably, a recent meta-analysis suggests that the supplementary motor area may mediate dlPFC emotion regulatory effects on the amygdala (Kohn et al, 2014). Finally, it is worth noting that, in the present study, the PTSD+DS group did not display increased connectivity between the BLA and the ACC, as we had hypothesized based on the model by Lanius et al (2010b). Future studies will therefore be required to determine whether the presence of an emotional stimulus (as opposed to resting state) will lead to altered amygdala-ACC connectivity in PTSD+DS. 


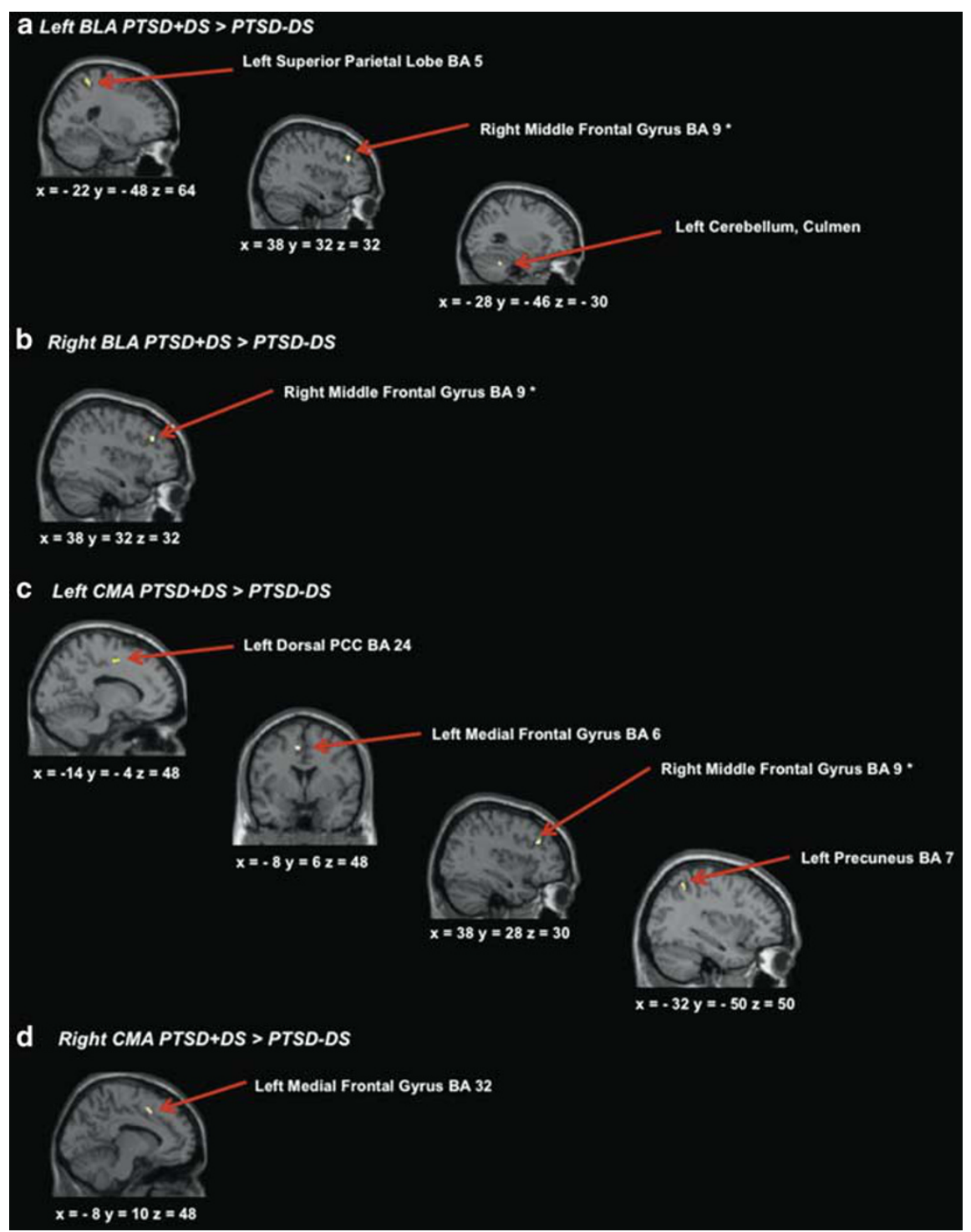

Figure I (a) Brain areas representing greater connectivity to the left basolateral amygdala within PTSD+DS as compared with PTSD - DS; (b) brain areas representing greater connectivity to the right basolateral amygdala within the PTSD+DS as compared with PTSD - DS; (c) brain areas representing greater connectivity to the left centromedial amygdala within PTSD+DS, as compared with PTSD - DS; (d) brain areas representing greater connectivity to the right centromedial amygdala within PTSD+DS as compared with PTSD - DS. Statistical threshold $p<0.005$ uncorrected, $k=10$ for all two-sample $t$-tests. BLA, basolateral amygdala; CMA, centromedial amygdala; PCC, posterior cingulate cortex; PTSD+DS, dissociative subtype posttraumatic stress disorder group; PTSD - DS, non-dissociative posttraumatic stress disorder group. *Indicates the a-priori region-of-interest analysis.

We had further hypothesized that there would be altered between-group connectivity with regard to the BLA and CMA and parietal regions responsible for regulating consciousness, awareness, and proprioception phenomena central to depersonalization and derealization experiences. The PTSD+DS group showed greater connectivity as compared with the PTSD - DS group between the left BLA and the left SPL. The SPL is involved in spatial orientation and is characterized by visual and sensory inputs (Wolbers et al, 2003), where damage to the SPL results in astereognosis - the inability to recognize objects through touch (Davis et al, 2006). The SPL also has a significant role in motor imagery, monitoring of imagined limb configuration, and proprioception (Wolbers et al, 2003). Increased connectivity between the BLA (which mediates cortical integration of fear and emotional responses) and the SPL may therefore be directly related to symptoms of depersonalization, where alterations in SPL function may occur during an out-of-body experience and would thus need to be integrated by the BLA.

The PTSD+DS group showed greater connectivity between the left CMA and the precuneus as compared with the PTSD - DS group. The precuneus has been shown to be involved in first-person perspective taking, consciousness, and self-related mental representations during rest (Cavanna and Trimble, 2006). This finding parallels our hypotheses, where those functions subtending consciousness and formation of perspective are likely altered in depersonalization responses, characteristic of PTSD+DS. In support of this finding, Medford et al (2006) report altered precuneus activity in depersonalization disorder 
Table 4 Dissociative Subtype and Non-dissociative subtype PTSD Between-Group Connectivity Differences

\begin{tabular}{|c|c|c|c|c|c|c|c|c|c|c|c|c|c|}
\hline \multirow[t]{2}{*}{ Seed region } & \multirow[t]{2}{*}{ Contrast } & \multirow[t]{2}{*}{ Gyrus/sulcus } & \multirow[t]{2}{*}{$\mathbf{H}$} & \multirow[t]{2}{*}{ BA } & \multirow{2}{*}{$\begin{array}{c}\text { Cluster } \\
\text { size }\end{array}$} & \multicolumn{2}{|c|}{ Beta values } & \multicolumn{3}{|c|}{ MNI coordinate } & \multirow{2}{*}{$\stackrel{t}{t}$} & \multirow{2}{*}{$\begin{array}{c}Z- \\
\text { score }\end{array}$} & \multirow[t]{2}{*}{ p Unc. } \\
\hline & & & & & & PTSD - DS & PTSD+DS & $\mathbf{x}$ & $\mathbf{Y}$ & $\mathbf{z}$ & & & \\
\hline \multirow[t]{3}{*}{ Left BLA } & PTSD - DS > PTSD+DS & NS & & & & & & & & & & & \\
\hline & PTSD - DS < PTSD+DS & Superior parietal lobe & $\mathrm{L}$ & 5 & 10 & 0.011 & 0.086 & -22 & -48 & 64 & 4.79 & 4.30 & $<0.0001$ \\
\hline & & Cerebellum, culmen & $L$ & & 10 & 0.043 & 0.115 & -28 & -46 & -30 & 3.23 & 3.06 & $=0.00 \mathrm{I}$ \\
\hline \multirow[t]{2}{*}{ Right BLA } & PTSD - DS > PTSD+DS & NS & & & & & & & & & & & \\
\hline & PTSD - DS < PTSD+DS & NS & & & & & & & & & & & \\
\hline \multirow[t]{4}{*}{ Left CMA } & PTSD - DS > PTSD+DS & NS & & & & & & & & & & & \\
\hline & PTSD - DS < PTSD+DS & Dorsal posterior cingulate & $L$ & 24 & 23 & -0.015 & 0.024 & -14 & -4 & 48 & 3.76 & 3.50 & $=0.0002$ \\
\hline & & Medial frontal gyrus & $\mathrm{L}$ & 6 & 23 & 0.003 & 0.053 & -8 & 6 & 48 & 3.00 & 2.90 & $=0.002$ \\
\hline & & Precuneus & $L$ & 7 & 18 & -0.012 & 0.055 & -32 & -50 & 50 & 3.09 & 3.00 & $=0.002$ \\
\hline \multirow[t]{2}{*}{ Right CMA } & PTSD - DS > PTSD+DS & NS & & & & & & & & & & & \\
\hline & PTSD - DS < PTSD+DS & Medial frontal gyrus & $L$ & 32 & 11 & -0.001 & 0.055 & -8 & 10 & 48 & 3.00 & 2.83 & $=0.002$ \\
\hline \multicolumn{14}{|l|}{ Table 4 (B) } \\
\hline \multirow[t]{2}{*}{ Seed region } & Contrast & Gyrus/sulcus & $\mathbf{H}$ & BA & $\begin{array}{c}\text { Cluster } \\
\text { size }\end{array}$ & \multicolumn{2}{|c|}{ Beta values } & \multicolumn{3}{|c|}{ MNI coordinate } & $t(47)$ & $\begin{array}{c}\text { Z- } \\
\text { score }\end{array}$ & p FWE \\
\hline & & & & & & PTSD - DS & PTSD+DS & $\mathbf{x}$ & $\mathbf{Y}$ & $\mathbf{z}$ & & & \\
\hline \multirow[t]{2}{*}{$\begin{array}{l}\text { Left BLA } \\
\end{array}$} & PTSD - DS > PTSD+DS & NS & & & & & & & & & & & \\
\hline & PTSD - DS < PTSD+DS & Middle frontal gyrus & $\mathrm{R}$ & 9 & 17 & 0.003 & 0.106 & 38 & 32 & 32 & 3.50 & 3.28 & $=0.042$ \\
\hline \multirow[t]{2}{*}{ Right BLA } & PTSD - DS > PTSD+DS & NS & & & & & & & & & & & \\
\hline & PTSD - DS < PTSD+DS & Middle frontal gyrus & $\mathrm{R}$ & 9 & 13 & -0.020 & 0.114 & 38 & 32 & 32 & 3.42 & 3.21 & $=0.045$ \\
\hline \multirow[t]{2}{*}{ Left CMA } & PTSD - DS > PTSD+DS & NS & & & & & & & & & & & \\
\hline & PTSD - DS <PTSD+DS & Middle frontal gyrus & $\mathrm{R}$ & 9 & 20 & 0.015 & 0.071 & 38 & 28 & 30 & 3.37 & 3.17 & $=0.041$ \\
\hline \multirow[t]{2}{*}{ Right CMA } & PTSD - DS > PTSD+DS & NS & & & & & & & & & & & \\
\hline & PTSD - DS <PTSD+DS & NS & & & & & & & & & & & \\
\hline \multicolumn{14}{|c|}{$\begin{array}{l}\text { Abbreviations: BA, Broadmann area; BLA, basolateral amygdala; CMA, centromedial amygdala; FWE, family-wise error corrected; H, hemisphere; PTSD - DS }>\text { PTSD+DS, contrast images representing greater connectivity in } \\
\text { the non-dissociative PTSD group compared with the dissociative subtype; PTSD - DS }<\text { PTSD+DS, contrast images representing greater connectivity in the dissociative subtype PTSD group compared with the non- } \\
\text { dissociative PTSD group; Unc., uncorrected for multiple comparisons. } \\
\text { (A) Follow-up comparisons from the ANOVA interaction, } p<0.005, k=10 \text {. } \\
\text { (B) Small volume correction a-priori analysis, FWE-corrected } p<0.05, k=10 \text {. }\end{array}$} \\
\hline
\end{tabular}


Table 5 Subscale MDI Depersonalization and Derealization Correlation to PTSD Amygdala Connectivity

\begin{tabular}{|c|c|c|c|c|c|c|c|c|c|c|}
\hline Seed region & \multirow[t]{2}{*}{ Gyrus/sulcus } & \multirow[t]{2}{*}{$\mathbf{H}$} & \multirow[t]{2}{*}{ BA } & \multirow[t]{2}{*}{ Cluster size } & \multicolumn{3}{|c|}{ MNI coordinate } & \multirow[t]{2}{*}{$t(47)$} & \multirow[t]{2}{*}{ Z-score } & \multirow[t]{2}{*}{ p Uncorrected } \\
\hline & & & & & $\mathbf{x}$ & $y$ & $\mathbf{z}$ & & & \\
\hline
\end{tabular}

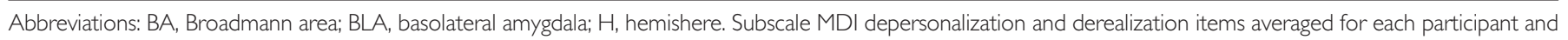
evaluated as a predictor of amygdala connectivity, including data from all PTSD (PTSD - DS and PTSD+DS) patients.

andicates the a-priori analysis.

patients as compared with controls when preforming an emotional verbal memory task.

The dorsal PCC also showed greater functional connectivity between the left CMA in the PTSD+DS group as compared with the PTSD - DS group. Generally, the PCC has been shown to be associated with visuospatial orientation that is mediated through its extensive parietal lobe connections and assessment of self-relevant sensations (Vogt and Laureys, 2005), and recently it has been suggested that the dorsal PCC is involved in conscious, awareness, and attention (Leech and Sharp, 2014). Moreover, the dorsal PCC is implicated in orienting the body toward innocuous and noxious somatosensory stimuli and mediates nociception, the encoding and processing of harmful stimuli (Vogt and Laureys, 2005). This also parallels previous structural findings suggesting the CMA to be implicated in major nociceptive pathways (Duvarci and Pare, 2014). Therefore, the PTSD+DS group may encode harmful stimuli and traumatic experiences in a unique, ie, detached way. Notably, the mid-cingulate cortex is very close to the dorsal PCC coordinate reported in the current study and is involved in reward value of behavioral outcomes during performance of cognitive tasks (Vogt and Laureys, 2005). Thus, through the encoding of reward, the brain may characterize dissociation as an adaptive response that mitigates overwhelming experience via emotional detachment. On balance, the current results suggest that the orchestration of fear responses via connectivity between the CMA and dorsal PCC may be unique in PTSD+DS patients, where self-relevant sensations, consciousness, awareness, and attention may be altered in dissociative states. These findings are consistent with results previously reported by Lanius et al (2005) in which PCC activity was greater in PTSD patients who showed depersonalization/derealization responses during traumatic recall relative to those PTSD patients who exhibited flashback/reliving symptoms.

Finally, the left BLA displayed increased connectivity between the culmen of the cerebellum in the PTSD+DS group as compared with the PTSD-DS group. The cerebellum has been previously shown to be involved in proprioception-the perception of limb configuration. Therefore, increased connectivity to the BLA may be important for integrating culmen functions of imagined limb configuration during PTSD+DS symptoms of depersonalization.

The between PTSD patient group comparisons in the current study revealed BLA connectivity to similar prefrontal brain regions reported by Brown et al (2014), albeit the current study does not report group differences to ACC, and Brown et al (2014) do not report any between-group differences in CMA connectivity. These connectivity differences between studies may be due to the use of trauma-exposed control participants in the study by Brown et al (2014) as opposed to healthy controls in the current study and/or to different types of trauma exposure (military $v s$ civilian). Furthermore, it is not clear whether the study by Brown et al (2014) included patients with PTSD+DS.

Several limitations of the current study are worth noting. Seed-based analyses have revealed significant sex-related differences in amygdala functional connectivity such that the amygdala exhibits greater functional connectivity at rest among females to the subgenual PFC and hypothalamus, when compared with males (Kilpatrick et al, 2006). Considering that the current sample is $75 \%$ female, the results may be biased towards the detection of differences in connectivity to prefrontal regions and may not be as sensitive to alterations in amygdala connectivity characteristic of males. In addition, the current study utilizes the DSM-IV CAPS as a diagnostic criteria for PTSD+DS. Subsequent studies should replicate the current findings using DSM-5 diagnostic criteria. Dynamic causal modeling should also be conducted in order to ascertain amygdala connectivity directionality in both the PTSD groups in larger samples. It is also critical to note that our analyses do not provide information on whether connectivity is inhibitory or excitatory. Moreover, cardiac and respiratory physiologicalrelated changes in BOLD signal were not corrected for. There is a mixed consensus in the field of functional connectivity regarding the need to correct for cardiac and respiratory physiological-related changes in BOLD signal (Birn et al, 2006; 2008; 2009; Birn, 2012; Cordes et al, 2001)—which is also reflected in the PTSD literature-where some studies remove physiological noise (eg, Roy et al, 2009; Sripada et al, 2012) and others do not (eg, Brown et al, 2014; Philip et al, 2013). Finally, future studies will need to examine how amygdala connectivity may change over the course of PTSD and also elucidate connectivity differences in response to emotional/traumatic triggers within the PTSD+DS and PTSD - DS groups.

In conclusion, the results of the current study point toward unique amygdala connectivity of both the BLA and CMA complexes among individuals with the dissociative subtype. Differences in amygdala complex connectivity to specific brain regions parallel unique symptoms of trauma within this PTSD group. Amygdala complex resting-state connectivity may serve as a biological marker for PTSD+DS, and the identification of specific patterns of BLA and CMA connectivity may help to further characterize the PTSD patient groups. Critically, these findings emphasize the importance of employing a heterogeneous conceptualization of the PTSD patient groups when designing neurobiological and clinical 
studies-as opposed to studying one amalgamated patient group. Moreover, our results may point to unique treatment avenues for various types of PTSD patients, such as using neurofeedback via real-time fMRI to mediate different connectivity patterns. Such studies are currently underway in our laboratory.

\section{FUNDING AND DISCLOSURE}

Funding for the current study was provided by the Canadian Institutes of Health Research (CIHR). The authors declare no conflict of interest.

\section{ACKNOWLEDGMENTS}

We thank Daniela Rabellino for her helpful comments on the manuscript.

\section{REFERENCES}

American Psychiatric Association (2013). Diagnostic and Statistical Manual of Mental Disorders, 5th edn. American Psychiatric Publishing: Washington, DC, USA.

Banks SJ, Eddy KT, Angstadt M, Nathan PJ, Phan KL (2007). Amygdala-frontal connectivity during emotion regulation. Soc Cogn Affect Neurosci 2: 303-312.

Beck AT, Guth D, Steer RA, Ball R (1997). Screening for major depression disorders in medical inpatients with the beck depression inventory for primary care. Behav Res Ther 35: 785-791.

Bernstein DP, Stein JA, Newcomb MD, Walker E, Pogge D, Ahluvalia T et al (2003). Development and validation of a brief screening version of the Childhood Truama Questionnaire. Child Abuse Neglect 27: 169-190.

Birn RM (2012). The role of physiological noise in resting-state functional connectivity. Neuroimage 62: 864-870.

Birn RM, Diamond JB, Smith MA, Bandettini PA (2006). Separating respiratory-variation-related fluctuations from neuronal-activityrelated fluctuations in fMRI. Neuroimage 31: 1536-1548.

Birn RM, Murphy K, Bandettini PA (2008). The effect of respiration variations on independent component analysis results of resting state functional connectivity. Human Brain Mapp 29: 740-750.

Birn RM, Murphy K, Handwerker DA, Bandettini PA (2009). fMRI in the presence of task-correlated breathing variations. NeuroImage 47: 1092-1104.

Birn RM, Patriat R, Phillips ML, Germain A, Herringa RJ (2014). Childhood maltreatment and combat posttraumatic stress differentially predict fear-related fronto-subcortical connectivity. Depress Anxiety 31: 880-892.

Blake DD, Weathers FW, Nagy LM, Kaloupek DG, Gusman FD, Charney DS et al (1995). The development of a ClinicianAdministered PTSD Scale. J Trauma Stress 8: 75-90.

Briere J, Weathers FW, Runtz M (2005). Is dissociation a multidimensional construct? Data from the multiscale dissociation inventory. J Trauma Stress 18: 221-231.

Brown VM, LaBar KS, Haswell CC, Gold AL, McCarthy G, Morey RA et al (2014). Altered resting-state functional connectivity of basolateral and centromedial amygdala complexes in posttraumatic stress disorder. Neuropsychopharmacology 39: 351-359.

Cavanna AE, Trimble MR (2006). The precuneus: a review of its functional anatomy and behavioural correlates. Brain 129: 564-583.

Cordes D, Haughton VM, Arfanakis K, Carew JD, Turski PA, Moritz CH et al (2001). Frequencies contributing to functional connectivity in the cerebral cortex in "resting-state" data. Am J Neuroradiol 22: 1326-1333.

Davis A, Whited A, Williams R, Pass L, Dean R, Hudson B (2006). Evaluating the presence of astereognosis in patients with multiple sclerosis. Arch Clin Neuropsychol 21: 519-519.

Duvarci S, Pare D (2014). Amygdala microcircuits controlling learned fear. Neuron 82: 966-980.

Eickhoff SB, Stephan KE, Mohlberg H, Grefkes C, Fink GR, Amunts $\mathrm{K}$ et al (2005). A new SPM toolbox for combining probabilistic cytoarchitectonic maps and functional imaging data. Neuroimage 25: 1325-1335.

Etkin A, Egner T, Kalisch R (2011). Emotional processing in anterior cingulate and medial prefrontal cortex. Trends Cogn Sci 15: 85-93.

Etkin A, Wager T (2007). Functional neuroimaging of anxiety: a meta-analysis of emotional processing in PTSD, social anxiety disorder, and specific phobia. Am J Psychiatry 164: 1476-1488.

Felmingham K, Kemp A, Williams L, Falconer E, Olivieri G, Peduto A et al (2008). Dissociative responses to conscious and non-conscious fear impact underlying brain function in posttraumatic stress disorder. Psychol Med 38: 1771-1780.

First MB (1997). User's Guide for the Structured Clinical Interview for DSM-IV Axis I Disorders SCID-I: Clinician Version. American Psychiatric Press: Washington, DC, USA.

Hopper JW, Frewen PA, van der Kolk BA, Lanius RA (2007). Neural correlates of reexperiencing, avoidance, and dissociation in PTSD: symptom dimensions and emotion dysregulation in responses to script-driven trauma imagery. J Trauma Stress 20: 713-725.

Jovanovic T, Ressler KJ (2010). How the neurocircuitry and genetics of fear inhibition may inform our understanding of PTSD. Am J Psychiatry 167: 648-662.

Kilpatrick LA, Zald DH, Pardo JV, Cahill LF (2006). Sex-related differences in amygdala functional connectivity during resting conditions. Neuroimage 30: 452-461.

Kohn N, Eickhoff SB, Scheller M, Laird AR, Fox PT, Habel U (2014). Neural network of cognitive emotion regulation - an ALE meta-analysis and MACM analysis. Neuroimage 87: 345-355.

Lanius RA, Bluhm RL, Coupland NJ, Hegadoren KM, Rowe B, Theberge J et al (2010a). Default mode network connectivity as a predictor of post-traumatic stress disorder symptom severity in acutely traumatized subjects. Acta Psychiatr Scand 121: 33-40.

Lanius RA, Brand B, Vermetten E, Frewen PA, Spiegel D (2012). The dissociative subtype of posttraumatic stress disorder: rationale, clinical and neurobiological evidence, and implications. Depress Anxiety 29: 701-708.

Lanius RA, Vermetten R, Loewenstein RJ, Brand B, Schmahl C, Bremner JD et al (2010b). Emotion modulation in PTSD: clinical and neurobiological evidence for a dissociative subtype. Am J Psychiatry 167: 640-647.

Lanius RA, Williamson PC, Bluhm RL, Densmore M, Boksman K, Neufeld RWJ et al (2005). Functional connectivity of dissociative responses in posttraumatic stress disorder: a functional magnetic resonance imaging investigation. Biol Psychiatry 57: 873-884.

Lanius RA, Williamson PC, Boksman K, Densmore M, Gupta M, Neufeld RWJ et al (2002). Brain activation during script-driven imagery induced dissociative responses in PTSD: a functional magnetic resonance imaging investigation. Biol Psychiatry 52: 305-311.

LeDoux J (1998). Fear and the brain: where have we been, and where are we going? Biol Psychiatry 44: 1229-1238.

Leech R, Sharp DJ (2014). The role of the posterior cingulate cortex in cognition and disease. Brain 137: 12-32.

Lévesque J, Eugène F, Joanette Y, Paquette V, Mensour B, Beaudoin G et al (2003). Neural circuitry underlying voluntary suppression of sadness. Biol Psychiatry 53: 502-510. 
Lieberman MD, Cunningham WA (2009). Type I and Type II error concerns in fMRI research: re-balancing the scale. Soc Cogn Affect Neurosci 4: 423-428.

Medford N, Brierley B, Brammer M, Bullmore ET, David AS, Phillips ML (2006). Emotional memory in depersonalization disorder: a functional MRI study. Psychiatry Res 148: 93-102.

Mickleborough MJ, Daniels JK, Coupland NJ, Kao R, Williamson PC, Lanius UF et al (2011). Effects of trauma-related cues on pain processing in posttraumatic stress disorder: an fMRI investigation. J Psychiatry Neurosci 36: 6-14.

Milad M (2013). Amygdala metabolism and associative fear learning in PTSD. Biol Psychiatry 73: 124S-124S.

Milad M (2014). Functional connectivity of the fear extinction network in PTSD patients using dynamic causal modeling. Biol Psychiatry 75: 13S-13S.

Milligan GW, Wong DS, Thompson PA (1987). Robustness properties of nonorthogonal analysis of variance. Psychol Bull 101: 464-470.

Phelps EA, Delgado MR, Nearing KI, LeDoux JE (2004). Extinction learning in humans: role of the amygdala and vmPFC. Neuron 43: 897-905.

Philip NS, Kuras YI, Valentine TR, Sweet LH, Tyrka AR, Price LH et al (2013). Regional homogeneity and resting state functional connectivity: associations with exposure to early life stress. Psychiatry Res 214: 247.

Roy AK, Shehzad Z, Margulies DS, Kelly AMC, Uddin LQ, Gotimer K et al (2009). Functional connectivity of the human amygdala using resting state fMRI. Neuroimage 45: 614-626.

Sadeh N, Spielberg JM, Warren SL, Miller GA, Heller W (2014). Aberrant neural connectivity during emotional processing associated with posttraumatic stress. Clin Psychol Sci 2: 748-755.

Shin LM, Liberzon I (2010). The neurocircuitry of fear, stress, and anxiety disorders. Neuropsychopharmacology 35: 169-191.

Shin LM, Wright CI, Cannistraro PA, Wedig MM, McMullin K, Martis B et al (2005). A functional magnetic resonance imaging study of amygdala and medial prefrontal cortex responses to overtly presented fearful faces in posttraumatic stress disorder. Arch Gen Psychiatry 62: 273-281.

Sripada RK, King AP, Garfinkel SN, Wang X, Sripada CS, Welsh RC et al (2012). Altered resting-state amygdala functional connectivity in men with posttraumatic stress disorder. J Psychiatry Neurosci 37: 241-249.

Stein DJ, Koenen KC, Friedman MJ, Hill E, McLaughlin KA, Petukhova $\mathrm{M}$ et al (2013). Dissociation in posttraumatic stress disorder: evidence from the World Mental Health Surveys. Biol Psychiatry 73: 302-312.

Steuwe C, Lanius RA, Frewen PA (2012). Evidence for a dissociative subtype of PTSD by latent profile and confirmatory factor analyses in a civilian sample. Depress Anxiety 29: 689-700.

Stevens JS, Jovanovic T, Fani N, Ely TD, Glover EM, Bradley B, Ressler KJ (2013). Disrupted amygdala-prefrontal functional connectivity in civilian women with posttraumatic stress disorder. J Psychiatric Res 47: 1469-1478.

Vogt BA, Laureys S. (2005). Posterior cingulate, precuneal and retrosplenial cortices: cytology and components of the neural network correlates of consciousness. Progr Brain Res 150: 205-217.

Wolbers T, Weiller C, Büchel C (2003). Contralateral coding of imagined body parts in the superior parietal lobe. Cereb Cortex 13: 392-399.

Wolf EJ, Miller M, Rearon AF, Rybchenko KA, Castillo D, Freund R (2012). Latent class analysis of dissociation and PTSD: evidence for a dissociative subtype. Arch Gen Psychiatry 69: 698-705.

Wolf EJ, Rasmusson AM, Mitchell KS, Logue MW, Baldwin CT, Miller MW (2014). A genome-wide association study of clinical symptoms of dissociation in a trauma-exposed sample. Depress Anxiety 31: 352-360.

Yan X, Lazar M, Shalev AY, Neylan TC, Wolkowitz OM, Brown AD et al (2014). Precuneal and amygdala spontaneous activity and functional connectivity in war-zone-re-lated PTSD. Psychiatric Res (e-pub ahead of print).

Supplementary Information accompanies the paper on the Neuropsychopharmacology website (http://www.nature.com/npp) 